\title{
Isolation and characterization of ZZ1, a novel lytic phage that infects Acinetobacter baumannii clinical isolates
}

Jing Jin ${ }^{1}$, Zhen-Jiang Li ${ }^{2}$, Shu-Wei Wang ${ }^{2}$, Shan-Mei Wang ${ }^{4}$, De-Hai Huang ${ }^{2}$, Ya-Hui Li ${ }^{2}$, Yun-Yun Ma², Jin Wang ${ }^{2}$, Fang Liu ${ }^{3}$, Xiang-Dong Chen ${ }^{3}$, Guang-Xing Li ${ }^{1}$, Xiao-Ting Wang ${ }^{1}$, Zhong-Quan Wang ${ }^{1^{*}}$ and Guo-Qiang Zhao ${ }^{1^{*}}$

\begin{abstract}
Background: Acinetobacter baumannii, a significant nosocomial pathogen, has evolved resistance to almost all conventional antimicrobial drugs. Bacteriophage therapy is a potential alternative treatment for multidrug-resistant bacterial infections. In this study, one lytic bacteriophage, ZZ1, which infects A. baumannii and has a broad host range, was selected for characterization.

Results: Phage ZZ1 and 3 of its natural hosts, A. baumanni clinical isolates AB09V, AB0902, and AB0901, are described in this study. The 3 strains have different sensitivities to ZZ1, but they have the same sensitivity to antibiotics. They are resistant to almost all of the antibiotics tested, except for polymyxin. Several aspects of the life cycle of ZZ1 were investigated using the sensitive strain AB09V under optimal growth conditions. ZZ1 is highly infectious with a short latent period (9 min) and a large burst size (200 PFU/cell). It exhibited the most powerful antibacterial activity at temperatures ranging from $35^{\circ} \mathrm{C}$ to $39^{\circ} \mathrm{C}$. Moreover, when ZZ1 alone was incubated at different $\mathrm{pHs}$ and different temperatures, the phage was stable over a wide $\mathrm{pH}$ range (4 to 9) and at extreme temperatures (between $50^{\circ} \mathrm{C}$ and $60^{\circ} \mathrm{C}$ ). ZZ1 possesses a 100-nm icosahedral head containing double-stranded DNA with a total length of 166,682 bp and a 120-nm long contractile tail. Morphologically, it could be classified as a member of the Myoviridae family and the Caudovirales order. Bioinformatic analysis of the phage whole genome sequence further suggested that ZZ1 was more likely to be a new member of the Myoviridae phages. Most of the predicted ORFs of the phage were similar to the predicted ORFs from other Acinetobacter phages.
\end{abstract}

Conclusion: The phage ZZ1 has a relatively broad lytic spectrum, high pH stability, strong heat resistance, and efficient antibacterial potential at body temperature. These characteristics greatly increase the utility of this phage as an antibacterial agent; thus, it should be further investigated.

Keywords: Acinetobacter baumannii , Bacteriophage, Characterization

\section{Background}

Bacteriophage therapy is one of the emerging methods used to overcome bacterial infections [1-3]. Bacteriophages are viruses that infect and kill bacteria. Theoretically, phages have several advantages over antibiotics. They are highly specific and very effectively lyse targeted pathogenic bacteria. They are safe because they have no activity against animal or plant cells. Phages are ubiquitous, so

\footnotetext{
* Correspondence: wangzq@zzu.edu.cn; zhaogq@zzu.edu.cn

'Department of Pathogen Biology, Basic Medical College of Zhengzhou

University, Kexue Road \# 100, Zhengzhou 450001, P. R. China

Full list of author information is available at the end of the article
}

isolation of new phages is a relatively rapid process that can frequently be accomplished in days or weeks. The use of phages as therapeutic agents was initiated in 1919, 3 years after their discovery, for the treatment of bacillary dysentery and continued until the 1940s. Over this time period, phages were used to treat a variety of infectious diseases [4]. However, with the advent of antibiotics, commercial production of therapeutic phages ceased in most of the Western world [5]. Currently, there is renewed interest in phage research and the applications of bacteriophages as potentially powerful antibacterial agents due to the emergence of drug-resistant pathogens and the dearth

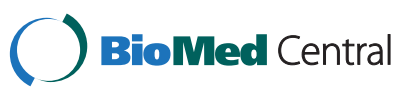

(c) 2012 Jin et al.; licensee BioMed Central Ltd. This is an Open Access article distributed under the terms of the Creative Commons Attribution License (http://creativecommons.org/licenses/by/2.0), which permits unrestricted use, distribution, and reproduction in any medium, provided the original work is properly cited. 
of new antibiotics. Several studies have shown that bacteriophages can be used successfully for therapeutic purposes, both in humans and animals [6-9]. However, more research is required before clinical use can be re-initiated. Before using a phage for therapeutic purposes, the isolation of lytic phages and characterization of the phage are essential.

In this study, clinical isolates of Acinetobacter baumannii were collected and used as indicator hosts to screen phages from water samples. A. baumannii mostly infects debilitated patients in intensive care units and is associated with high mortality rates $[10,11]$. Since its discovery, A. baumannii has become resistant to many common antibiotics [12]. The increasing prevalence of multidrugand pandrug-resistant $A$. baumannii strains in clinics has rendered it one of the most important nosocomial pathogens [12-15]. Fortunately, lytic phages specific to $A$. baumannii might provide an alternative to antibiotics for the prevention and treatment of infections caused by this bacterium. However, to the best of our knowledge, very few detailed characterizations of $A$. baumannii phages have been published [16,17]. This paper describes the isolation and characterization of a novel virulent phage, $\mathrm{ZZ1}$, that infects $A$. baumannii clinical isolates.

\section{Results}

Isolation of ZZ1 and its morphology

Twenty-three $A$. baumannii clinical isolates were screened for phage present in a sample of fishpond water. Among these, only the strain AB09V could serve as an indicator for ZZ1 in the initial screening. This phage formed clear plaques of approximately 1-2 $\mathrm{mm}$ in diameter on AB09V lawns. $\mathrm{AB} 09 \mathrm{~V}$ was thus used to propagate, purify and characterize the phage. As shown in Figure 1, the phage ZZ1 has a 100-nm icosahedral head and a 120-nm long contractile tail. Morphologically, phage ZZ1 can be tentatively classified as a member of the Myoviridae family in the order of Caudovirales. Most of the input phages rapidly adsorbed to AB09V cells. Appearance of ghost particles 5 min after mixing phages with bacteria suggested that ejection of DNA from the phage head occurred rapidly.

\section{Host range of $Z Z 1$ and identification of bacterial strains}

Two additional natural bacterial hosts, AB0901 and AB0902, were found when the other 22 of the $23 \mathrm{~A}$. baumannii clinical isolates were used to investigate the host range of $\mathrm{ZZ1}$ by spot test. This test used a higher concentration of phage $\left(10^{8} \mathrm{PFU} / \mathrm{ml}\right)$ than the original screen. Interestingly, some differences were observed in the ability of the phage to lyse the 3 bacterial hosts (AB09V, AB0901, and AB0902). For example, as shown Figure 2, ZZ1 was capable of forming transparent areas on lawns of the strains AB09V, AB0901, and AB0902. However, the minimum phage concentrations required to form clear spots on each lawn were different: AB09V required $10^{5} \mathrm{PFU} / \mathrm{ml}$, AB0902 required $10^{6} \mathrm{PFU} / \mathrm{ml}$, and AB0901 required $10^{8}$ $\mathrm{PFU} / \mathrm{ml}$. The values suggest that under the same culture conditions, the antibacterial activity of ZZ1 was highest in strain AB09V, followed by AB0902 and then AB0901. There might be natural resistance mechanisms in AB0901 and AB0902; thus, the strain AB09V is likely the most sensitive indicator of the phage titer of the 3 strains and is the best host for phage propagation.

The 3 strains that can be infected by ZZ1 were selected for further detailed study. They were resistant to all of the

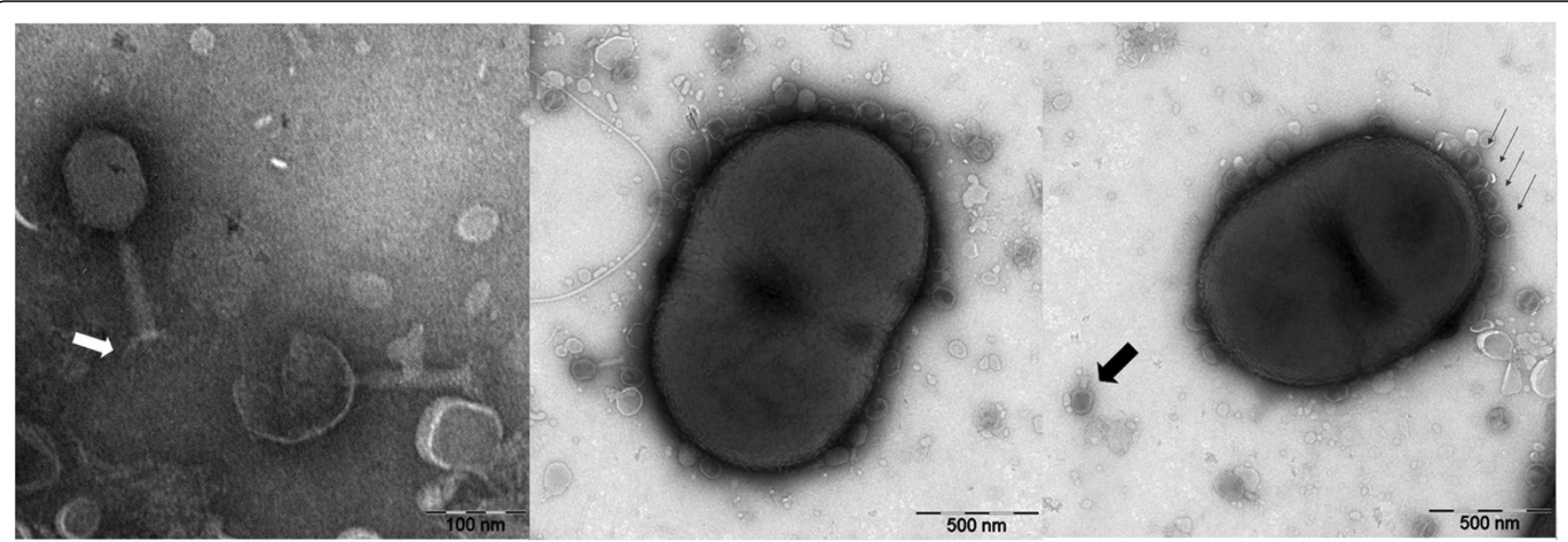

Figure 1 Electron micrographs of ZZ1 and infected A. baumannii AB09V. A mixture of ZZ1 phages and A. baumannii AB09V cells was negatively stained. The phage ZZ1 contained a baseplate with fibers (indicated by the white arrow) and an icosahedral head with a contractile tail (indicated by the large black arrow), which allowed for its inclusion in the Myoviridae family of the order Caudovirales. Intact phages had a head filled with DNA, and ghost particles (indicated by the small black arrows) had an empty head, showing that ejection of DNA from the phage head had taken place within 5 min. 


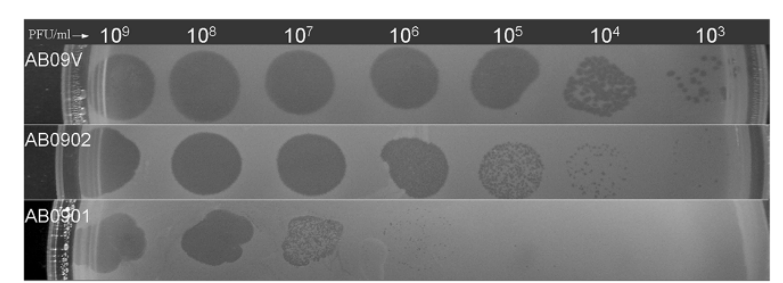

Figure 2 Antibacterial activity of phage $Z Z 1$ against three $A$. baumannii strains. Serial 10-fold dilutions of phage ZZ1 were spotted onto lawns of strains AB09V, AB0901, and AB0902 in 0.7\% agar nutrient broth at $37^{\circ} \mathrm{C}$. AB09V was used as the indicator for determination of the phage titer.

antibiotics tested except polymyxin (amikacin, gentamicin, imipenem, meropenem, cefazolin, ceftazidime, cefotaxime, cefepime, aztreonam, ampicillin, piperacillin, amoxicillin/ clavulanic acid, ampicillin/sulbactam, piperacillin/tazobactam, sulfanilamide, sulfamethoxazole and trimethoprim, ciprofloxacin, levofloxacin, and tetracycline). Partial $16 \mathrm{~S}$ rRNA genes of the 3 strains were sequenced and deposited in GenBank under the accession numbers [GenBank: JF313142] (AB09V), [GenBank: JF313143] (AB0901), and [GenBank: JF313144] (AB0902). Nucleotide blast analysis further confirmed that the three strains were $A$. baumannii.

\section{Stability investigation}

Temperature and $\mathrm{pH}$ stability are two important parameters in the storage of therapeutic phage. Thus, the stability of ZZ1 was investigated at different $\mathrm{pHs}$ and temperatures. As shown in Figure 3, no obvious effect on ZZ1 activity was observed after $1 \mathrm{~h}$ of incubation at $\mathrm{pH}$ levels ranging from 4 to 9 . However, the phage completely lost its infectivity at $\mathrm{pH} 10$ or higher and

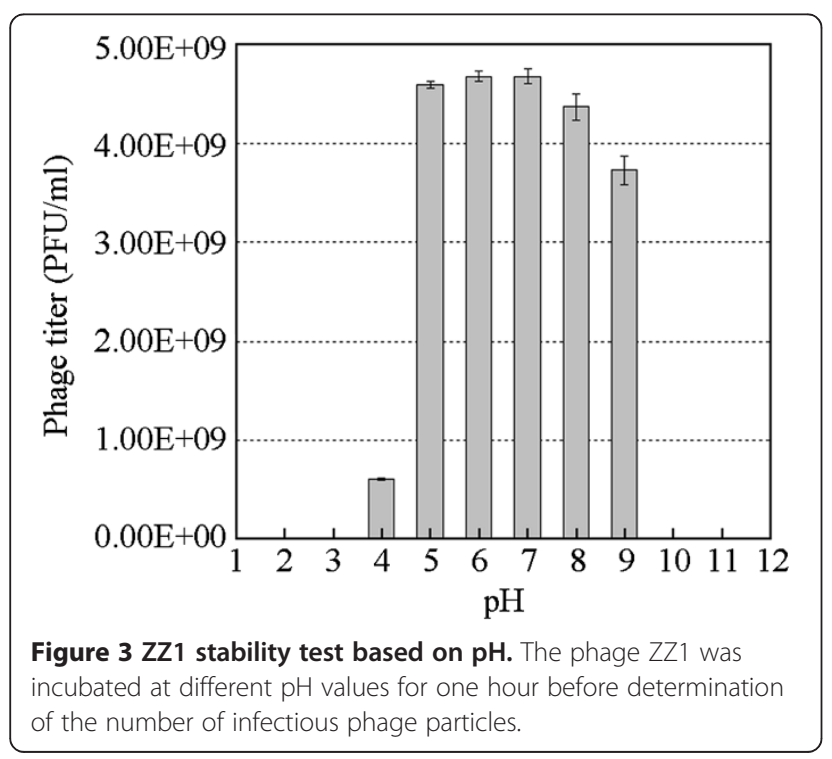

$\mathrm{pH} 3$ or lower. Within $1 \mathrm{~h}$ of incubation at $\mathrm{pH} 4$, the phage infectivity decreased by approximately $87 \%$, and a titer of $6.0 \times 10^{8} \mathrm{PFU} / \mathrm{ml}$ of active phage $\mathrm{ZZ1}$ was detected at the end of the incubation. The maximum stability of the phage was observed at a $\mathrm{pH}$ between 6 and 7. In addition, the results of thermal stability tests shown in Figure 4 suggest that ZZ1 was relatively heat stable over $1 \mathrm{~h}$ at temperatures between $50^{\circ} \mathrm{C}$ and $60^{\circ} \mathrm{C}$, and no significant loss in phage activity was observed. At $70^{\circ} \mathrm{C}$, the phage titer quickly dropped, and no viral particles were detected after $40 \mathrm{~min}$. Furthermore, phage activity was completely lost at $80^{\circ} \mathrm{C}$ within the first $1 \mathrm{~min}$ of incubation. The $\mathrm{ZZ1}$ phage lysate retained almost $100 \%$ of its infection activity when stored at both $25^{\circ} \mathrm{C}$ and $4^{\circ} \mathrm{C}$ for several months (data not shown).

\section{Investigation of antimicrobial activity of ZZ1 against $\mathrm{AB09V}$ at different temperatures}

Optimal A. baumannii growth occurs over a very broad temperature range [10]. As shown in Figure 5, the AB09V lawns grew well on nutrient agar plates at temperatures ranging from $25^{\circ} \mathrm{C}$ to $42^{\circ} \mathrm{C}$. However, the antimicrobial activity of $\mathrm{ZZ1}$ is clearly influenced by temperature variations. When the plates were incubated at different temperatures, the minimum phage concentrations required to form clear spots on AB09V lawns were different: $10^{5} \mathrm{PFU} / \mathrm{ml}$ at $35^{\circ} \mathrm{C}, 37^{\circ} \mathrm{C}$, and $39^{\circ} \mathrm{C}$; $10^{6} \mathrm{PFU} / \mathrm{ml}$ at $30^{\circ} \mathrm{C}$ and $40^{\circ} \mathrm{C} ; 10^{8} \mathrm{PFU} / \mathrm{ml}$ at $25^{\circ} \mathrm{C}$; and $10^{9} \mathrm{PFU} / \mathrm{ml}$ at $42^{\circ} \mathrm{C}$. Thus, we concluded that $\mathrm{ZZ1}$ exhibits the most efficient antibacterial activity against the $\mathrm{AB} 09 \mathrm{~V}$ strain at temperatures ranging from $35^{\circ} \mathrm{C}$ to $39^{\circ} \mathrm{C}$, which suggested that ZZ1 could exhibit powerful antibacterial activity against AB09V at body temperature.

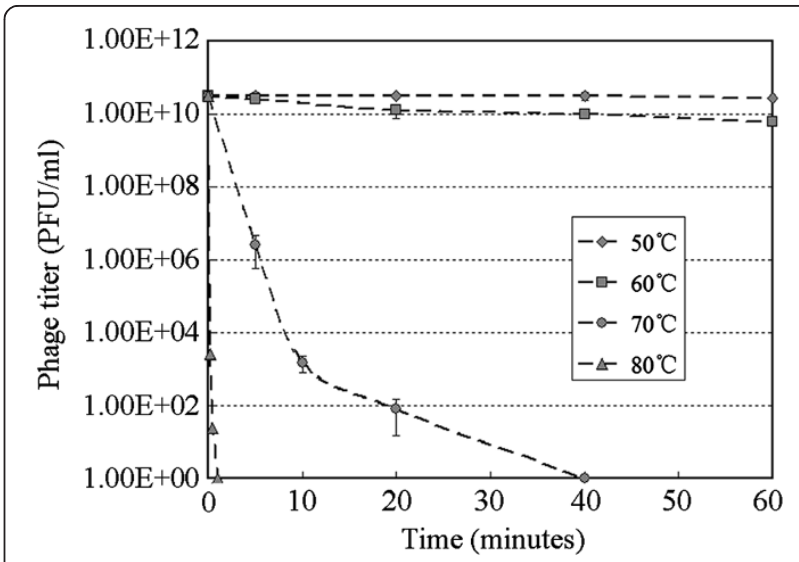

Figure 4 ZZ1 heat stability test. Samples were taken at different time intervals to determine the titer of the surviving infectious phage particles. 


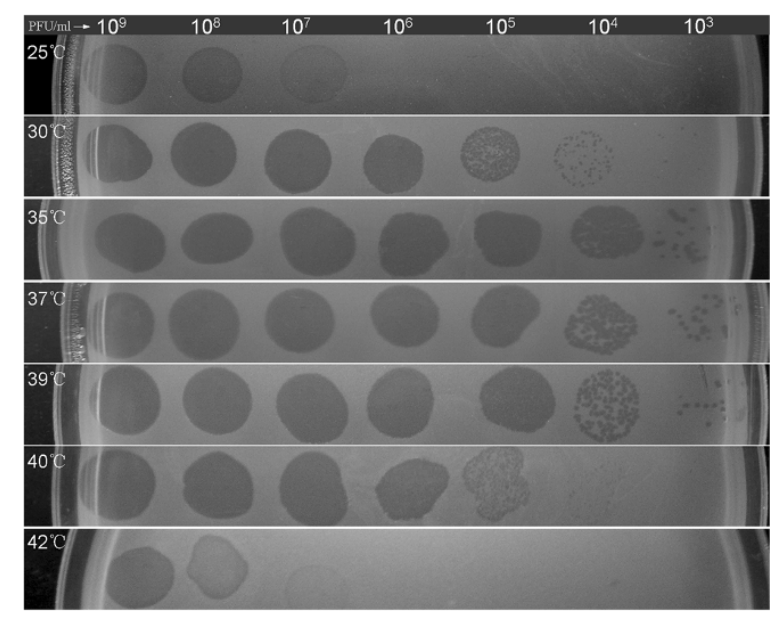

Figure 5 Optimal temperature for antibacterial activity of ZZ1 against $A$. baumannii AB09V. Serial 10-fold dilutions of phage ZZ1 were spotted onto lawns of the sensitive strain AB09V in 0.7\% agar nutrient broth at different temperatures.

\section{Phage growth attributes on $A B 09 \mathrm{~V}$}

The growth characteristics of ZZ1 on the sensitive indicator strain $\mathrm{AB} 09 \mathrm{~V}$ were characterized under optimal growth conditions. Phage ZZ1 exhibited high infection efficiency after mixing the phages and AB09V cells. We inferred that almost all of the A. baumannii AB09V were infected prior to the burst time of the first infected cell because the number of bacteria surviving at 9 min was less than $100 \mathrm{CFU} / \mathrm{ml}$. Moreover, as shown in Figure 6, the total plaque count was $6.6 \times 10^{8} \mathrm{PFU} / \mathrm{ml}$ at the beginning of infection $(0 \mathrm{~min})$, and only $2.3 \times 10^{8} \mathrm{PFU} / \mathrm{ml}$ remained after $9 \mathrm{~min}$. The difference (approximately $4.3 \times 10^{8} \mathrm{PFU} /$ $\mathrm{ml}$ ) originated from adsorption of multiple phage particles to one susceptible bacterial cell. The decrease in the number of phages was greater than 6 -fold higher than the initial number of bacterial cells (approximately $7 \times 10^{7} \mathrm{CFU} /$ $\mathrm{ml})$. These results further confirmed that almost all of the bacterial cells could be infected within the latent period (9 min). The number of unattached phages at the end of the latent period (or prior to the burst time of the first infected cells) can be estimated as the difference between the number of the total plaque count and the initial number of bacterial cells. The calculated number of unattached phages was $1.6 \times 10^{8} \mathrm{PFU} / \mathrm{ml}$, which is negligible compared to the phage number at the end of the experiment $\left(1.5 \times 10^{10} \mathrm{PFU} / \mathrm{ml}\right)$. Moreover, the number of bacteria surviving at the end of the experiment is less than $50 \mathrm{CFU} / \mathrm{ml}$, which can also be considered negligible when compared to the initial number of bacterial cells $\left(7.0 \times 10^{7} \mathrm{CFU} / \mathrm{ml}\right)$. Therefore, the average burst size was approximately $200 \mathrm{PFU} /$ cell, which can be calculated as the ratio of the final count of phage particles to the initial count of infected bacterial cells.

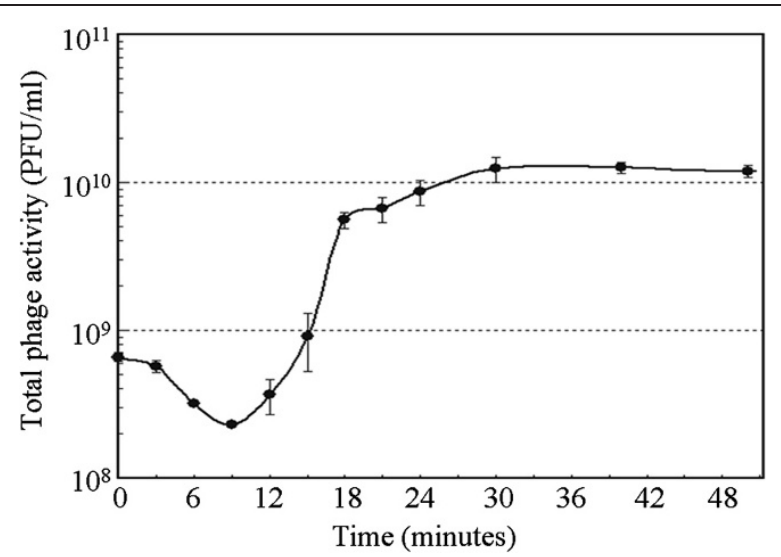

Figure 6 One-step growth curve of ZZ1 on A. baumannii AB09V. Phage ZZ1 was mixed with strain AB09V at an MOI of approximately 10 at $37^{\circ} \mathrm{C}$ (The initial ratio of phage concentration to bacterial concentration is $\left.6.6 \times 10^{8} \mathrm{PFU} / \mathrm{ml}: 7.0 \times 10^{7} \mathrm{CFU} / \mathrm{ml}\right)$. Then, the total phage activity (including infected bacterial cells and free phages) was determined periodically. The decline in the concentration of total phages occurred as a result of the binding of multiple viral particles to one susceptible bacterial cell followed by a rapid increase, resulting in release of phages by lysis of the infected bacterial cells. The ZZ1 latent period was approximately $9 \mathrm{~min}$, and the burst size averaged 200 PFU per infected cell.

\section{Genome analysis}

The phage nucleic acid was susceptible to degradation by DNase and some restriction endonucleases (e.g., HindIII, EcoRI, and EcoRV) but unaffected by RNase. Thus, ZZ1 is a dsDNA phage (data not shown). The ZZ1 genome has a total length of 166,682 bp and a GC content of $34.3 \%$, which is slightly lower than that described for the A. baumannii ATCC 17978 strain (38\%, accession number NC_009085). An initial NCBI nucleotide blast analysis (blastn) of the complete genome sequence indicated that ZZ1 shares limited similarities with other known phage nucleotide sequences, which confirmed its status as a novel Acinetobacter phage species. The top 4 most similar sequences found were of the Acinetobacter phages Acj9 [GenBank: HM004124.1], Acj61 [GenBank: GU911519.1], Ac42 [GenBank: HM032710.1], and 133 [GenBank: HM114315.1]. The max scores were 4662 (50\% of coverage, $89 \%$ of max ident), 4448 (45\% of coverage, $87 \%$ of max ident), 2634 (34\% of coverage, $94 \%$ of max ident), and 2210 (31\% of coverage, $92 \%$ of max ident). The four Acinetobacter phages were recently deposited in GenBank and were previously annotated as T4-like phages [18]. No other Acinetobacter phages were hit by blastn. In addition, Enterobacteria phage T4 ranked tenth, and its max score was 1972 (28\% of coverage, $83 \%$ of max ident), suggesting that the ZZ1 phage might be a new member of the T4-like phage family.

A sequence search using the NCBI open reading frame (ORF) finder revealed a total of 402 putative ORFs of 50 
or more codons in the ZZ1 genome that have limited similarity to other known phage proteins. Among them, 118 ORFs have the highest similarity to predicted ORFs from the Acinetobacter phage Acj9; 47 ORFs are most similar to predicted ORFs from the Acinetobacter phage Acj61; 18 ORFs most closely resemble predicted ORFs from the Acinetobacter phage 133; and only 13 ORFs have the highest score with predicted ORFs from the Acinetobacter phage Ac42. In addition, of the 402 ORFs, 105 ORFs showed homology with sequences in GenBank with annotated function; 244 ORFs had matches with uncharacterized entries; and the remaining 53 ORFs had no match to sequenced genes in the database.

\section{Discussion}

Phage therapy has been the subject of several recent reviews, and the present study reinforces the view that it is worth exploring $[1,2,19]$. To the best of our knowledge, the characterization of lytic phages of A. baumannii has rarely been studied, although Ackermann et al. $[16,20]$ described the classification of an A. baumannii phage, and Soothill et al. [1,21] tested the efficacy of phage therapy for experimental $A$. baumannii infections in mice. In this study, we focused our efforts on the isolation and characterization of $A$. baumannii phages with potential for prophylactic/therapeutic use.

Phages are thought to be found wherever bacteria thrive [22]. Acinetobacter spp. are ubiquitous organisms that can be readily isolated from non-clinical sources, such as soil, drinking and surface waters, sewage, and a variety of different foods [10], which suggests that phages specific to A. baumannii might also be easily isolated from nature. Recently, 10 phages were obtained from wastewater using 125 clinical isolates of $A$. baumannii as indicator hosts [20,23]. These phages were designated $\mathrm{AB} 1$ to $\mathrm{AB} 9$ and $\mathrm{AB} 11$. Examination by transmission electron microscopy suggested that phages AB1-7 and AB9 belonged to the Podoviridae family, and phages AB8 and AB11 belonged to the Myoviridae family. Two of the 10 phages, $A B 1$ and $A B 2$, were further characterized at $35^{\circ} \mathrm{C}$ and $37^{\circ} \mathrm{C}$, respectively. Based on morphology and genomic analysis, the two phages were classified as new members of the $\phi K M V$-like phages $[20,23]$. In this study, the phage $Z Z 1$, which is specific to $A$. baumannii, was isolated from fishpond water, which further confirmed that phages specific to A. baumannii are waiting to be exploited as an abundant natural resource. The ability of phage ZZ1 to form clear plaques on lawns of $\mathrm{AB} 09 \mathrm{~V}$ is indicative of lytic phage, and a large burst size with a short latent period is further suggestive of the lytic nature of phage ZZ1. Morphologically, ZZ1 exhibits features similar to the Myoviridae family (order Caudovirales), which, broadly, are tailed phages with icosahedral head symmetry and contractile tail structures. Genome analysis of ZZ1 showed that it is fairly similar to four other Acinetobacter phages (Acj9, Acj61, Ac42, and 133). In a recent review by Petrov et al. [18], the four Acinetobacter phages were initially assigned to the "T4-like Viruses" genus. Each of these Acinetobacter phages has a unique set of ORFs that occupy $\sim 35 \%$ of the genome. That is, each represents a different type of T4-related phage genome [18]. The genome size of the phage ZZ1 (166,682 bp) is also close to the genome size of T4-like phages. These genomes vary between $\sim 160,000$ and $\sim 250,000$ bp [18]. Therefore, the above features confirmed that the phage ZZ1 is most likely a new member of the T4-like virus family of Acinetobacter phages. However, according to the 2011 Virus Taxonomy List (current) from the International Committee for the Taxonomy of Viruses (http://www.ncbi. nlm.nih.gov/ICTVdb/index.htm.), only the Acinetobacter phage 133 can be searched and classified in the unassigned genus of the Myoviridae family, most likely because the phage is inadequately characterized. At the very least, the current sequence database for the Myoviridae phages should prove to be a rich source of genetic markers for bioprospecting and a mine of reagents for basic research and biotechnology. Our future research will focus on further detailed analysis of the whole ZZ1 genome to understand the genetic characteristics of this phage.

The main aim of this study was the isolation and characterization of a lytic bacteriophage with potential for prophylactic/therapeutic use. Therefore, the antibacterial activity of the phage against its different host cells was the focus of our research. Through the preliminary in vitro host range investigation, ZZ1 was found to have different antibacterial activity against 3 of its natural hosts. The antibacterial activity of ZZ1 was highest against the strain AB09V, followed by AB0902 and then AB0901, based on the minimum phage concentration required to form clear spots at $37^{\circ} \mathrm{C}$. The natural resistance mechanisms of AB0901 and AB0902 against ZZ1 are worth further investigation in future studies. With respect to its life cycle in the sensitive strain AB09V, ZZ1 proliferates efficiently, with a short latent period ( $9 \mathrm{~min})$, a large burst size (200 $\mathrm{PFU} / \mathrm{ml}$ ), and a high adsorption rate. Remarkably, only less than $50 \mathrm{CFU} / \mathrm{ml}$ of the AB09V cells remained viable 30 min after $A B 09 V$ cells were mixed with $\mathrm{ZZ1}$ particles at a multiplicity of infection (MOI) of 10 at $37^{\circ} \mathrm{C}$. Moreover, ZZ1 exhibited the most powerful antibacterial activity at temperatures ranging from $35^{\circ} \mathrm{C}$ to $39^{\circ} \mathrm{C}$, suggesting that the phage would be highly effective when placed inside the body at normal or near normal body temperature. In addition, $\mathrm{ZZ1}$ was stable over a wide $\mathrm{pH}$ range (4-9) and was strongly resistant to heat. All of these features have implications for the use of this phage as a stable therapeutic agent for the treatment of $A$. baumannii 
infections, especially those caused by the strain most sensitive to the phage, AB09V. The differences in the antibacterial activity of ZZ1 against the strains tested will be the focus of our future research both in vitro and in vivo.

\section{Conclusions}

This study provides information about a novel virulent A. baumannii phage. Our future research will examine the application of this characterized phage in treating infections by $A$. baumannii clinical isolates both in vivo and in vitro.

\section{Methods}

\section{Bacterial strains and Identification}

Twenty-three clinical strains of $A$. baumannii were used in this study for phage isolation and phage host investigation. All of these strains were isolated from the sputum of hospitalized patients at the Henan Province People's Hospital in Zhengzhou, China. After obtaining the approval of the Life Science Ethics Committee of Zhengzhou University and written informed consent, sputum samples were collected for the purposes of this study. The automated system BD Phoenix (Becton Dickinson Diagnostic Systems, Sparks, MD, USA) was used on clinical samples for the identification of bacteria and for antibiotic susceptibility tests. Only 3 of the 23 strains could be lysed by ZZ1; these were lysed to varying degrees. Therefore, the 3 strains were designated AB09V, AB0901, and AB0902 in our nomenclature. The 3 strains selected for use in this study were further confirmed as A. baumannii using sequence information derived from their $16 \mathrm{~S}$ rRNA gene. Briefly, bacterial DNA was isolated as previously described [24]. The extracted DNA was used as the PCR template to amplify the $16 \mathrm{~S}$ ribosomal RNA coding regions. The ClustalX 2.0 program and Oligo 4.0 primer analysis software were used for universal primer design based on homology profiles among the $16 \mathrm{~S}$ rRNA genes of $A$. baumannii strains reported in the GenBank sequence database. The universal primers $199 \mathrm{f}$ (5' CTA CGG GAG AAA GCA GGG GAT 3') and 1344r (5' TTA CTA GCG ATT CCG ACT TCA 3') were used to amplify partial $16 \mathrm{~S}$ rRNA gene sequences. To increase the specificity of amplification and to reduce the formation of spurious byproducts, a "touchdown" PCR was performed (the annealing temperature decreased from 65 to $55^{\circ} \mathrm{C}$ for 20 cycles) as described previously [24]. The PCR amplicons were purified with a CONCERT Rapid PCR purification kit (Invitrogen) and were then sequenced directly with the primers.

\section{Bacteriophage isolation and growth}

Phage isolation was conducted using the method described by Adams [25]. Several water samples (municipal sewage, fishpond water, and river water) collected from different places in Zhengzhou, China, were clarified by centrifugation $\left(12,000 \times g\right.$ for $15 \mathrm{~min}$ at $\left.4^{\circ} \mathrm{C}\right)$. One percent $(\mathrm{v} / \mathrm{v})$ of a bacterial broth culture (overnight growth) along with an equal volume of nutrient broth at double concentration was added to the cleared supernatant and incubated at $37^{\circ} \mathrm{C}$ overnight. The next day, after centrifugation $\left(12,000 \times g\right.$ for $20 \mathrm{~min}$ at $\left.4^{\circ} \mathrm{C}\right)$, the supernatant was filtered with a $0.45 \mu \mathrm{m}$ SFCA Corning syringe filter (Corning Inc., Corning, NY) to remove the residual bacterial cells. An aliquot $(0.2 \mathrm{ml})$ of the filtrate was mixed with $0.1 \mathrm{ml}$ of an overnight culture of an A. baumannii strain and $2.5 \mathrm{ml}$ of molten top soft nutrient agar $(0.7 \%$ agar) at $47^{\circ} \mathrm{C}$ then overlaid on the surface of solidified base nutrient agar $(1.5 \%$ agar $)$ at $37^{\circ} \mathrm{C}$. After incubation overnight at $37^{\circ} \mathrm{C}$, the phage plaques were picked from the plates, and each individual plaque was re-isolated three times to ensure the purity of the phage isolate [26]. The phage titer was determined by the double-layered method [25].

Phage stocks were prepared on the most sensitive bacterial host using the soft layer plaque technique. Briefly, $10 \mathrm{ml}$ of an overnight $\mathrm{AB} 09 \mathrm{~V}$ bacterial culture was concentrated to $1 \mathrm{ml}$ by centrifugation $(3,000 \times g$ for $10 \mathrm{~min})$. One hundred microliters of the concentrated culture $\left(10^{10} \mathrm{CFU} / \mathrm{ml}\right)$ and $0.1 \mathrm{ml}$ of the phage $\mathrm{ZZ1}\left(10^{7} \mathrm{PFU} / \mathrm{ml}\right)$ were added to $2.5 \mathrm{ml}$ of molten top soft nutrient agar ( $0.4 \%$ agar) then overlaid on the surface of solidified base nutrient agar (1.5\% agar). The plates were incubated for 6$8 \mathrm{~h}$ at $37^{\circ} \mathrm{C}$ and were used to prepare a concentrated phage suspension $\left(10^{11} \mathrm{PFU} / \mathrm{ml}\right)$ by eluting the top agar overlaid plates in $5 \mathrm{ml} \mathrm{SM}$ buffer. Phage stocks were stored at $4^{\circ} \mathrm{C}$ after filtration through $0.45-\mu \mathrm{m}$ filters.

\section{Host range investigation}

The host range of the phages was examined by spot tests on 23 A. baumannii clinical strains. A $0.1 \mathrm{ml}$ aliquot of bacterial overnight broth culture $\left(10^{9} \mathrm{CFU} / \mathrm{ml}\right)$ was mixed with melted $0.7 \%$ soft nutrient agar $\left(47^{\circ} \mathrm{C}\right)$, and this mixture was poured onto $1.5 \%$ solid agar to make double layer ager plates. When the top agar hardened, phage stock $(5 \mu \mathrm{l})$ from a dilution series was spotted on each plate with different bacterial strains. The plates were incubated at $37^{\circ} \mathrm{C}$. When clearing zones were observed, the antibacterial activity of the phages against each bacterial host was assessed based on the minimum phage concentration required to form a completely transparent zone.

\section{Investigation of $Z Z 1$ antimicrobial activity against $A B 09 \mathrm{~V}$ at different temperatures}

The antibacterial activity of ZZ1 against A. baumannii AB09V was evaluated by serial dilution spot testing at different temperatures. Phage stock $(5 \mu \mathrm{l})$ from a dilution series was spotted onto a lawn of AB09V in top agar. The 
plates were examined for cell lysis after overnight incubations at $25^{\circ} \mathrm{C}, 30^{\circ} \mathrm{C}, 35^{\circ} \mathrm{C}, 37^{\circ} \mathrm{C}, 39^{\circ} \mathrm{C}, 40^{\circ} \mathrm{C}$, and $42^{\circ} \mathrm{C}$. The optimal antibacterial temperature was determined by comparing the minimum phage concentration required to form a completely transparent zone.

\section{Phage adsorption and growth curve}

An overnight culture of strain AB09V (1 ml) was inoculated into fresh medium $(100 \mathrm{ml})$ and incubated with shaking at $37^{\circ} \mathrm{C}$ for approximately $1 \mathrm{~h}$ to yield a cell density of approximately $7.0 \times 10^{7} \mathrm{CFU} / \mathrm{ml}$ (at an $\mathrm{OD}_{600}$ of 0.15). A $1 \mathrm{ml}$ sample of a nutrient broth suspension of the phage ZZ1 at an approximate MOI of 10 was added to this culture. Samples were periodically withdrawn and immediately chilled while being further diluted to measure total phage activity (including infected bacterial cells and free phages) by the doublelayered-agar plate technique. Bacterial viable counts were determined before the bacteria were mixed with the phage and were assessed periodically. Burst size was estimated from triplicate experiments using the equation described by Jiang et al. [27]. Each experiment was performed three times, and the results are reported as the mean of three observations \pm standard deviation (SD).

\section{Stability}

Resistance to different $\mathrm{pH}$ values at $37^{\circ} \mathrm{C}$ was determined according to the methods described by Verma et al. [28]. The $\mathrm{pH}$ of the nutrient broth was adjusted with either $1 \mathrm{M} \mathrm{HCl}$ or $1 \mathrm{M} \mathrm{NaOH}$ to obtain a pH within the range of $2-11$. A total of $100 \mu \mathrm{l}$ of bacteriophage suspension $\left(4.7 \times 10^{11} \mathrm{PFU} / \mathrm{ml}\right)$ was inoculated into $10 \mathrm{ml}$ of $\mathrm{pH}$-adjusted medium. After incubation for $1 \mathrm{~h}$ at $37^{\circ} \mathrm{C}$, the surviving phages were diluted and counted immediately using the soft agar overlay method at $37^{\circ} \mathrm{C}$. Moreover, according to the methods described by Capra et al. [29], the stability of ZZ1 at various temperatures $\left(50^{\circ} \mathrm{C}, 60^{\circ} \mathrm{C}, 70^{\circ} \mathrm{C}\right.$, and $\left.80^{\circ} \mathrm{C}\right)$ was checked by incubating the phage $\left(3.2 \times 10^{10} \mathrm{PFU} / \mathrm{ml}\right)$ at the indicated temperature for $1 \mathrm{~h}$ at $\mathrm{pH} 7.0$ in nutrient broth; the surviving phages were then counted using the soft agar overlay method at $37^{\circ} \mathrm{C}$.

\section{Morphology of phage and its host strain}

AB09V cells were infected with ZZ1 during the exponential growth phase $\left(\mathrm{OD}_{600}=0.35\right)$ at an MOI of approximately 100 and incubated at $37^{\circ} \mathrm{C}$ for 5 min in nutrient broth medium. The mixture was fixed with $1 \%$ glutaraldehyde at $0^{\circ} \mathrm{C}$ for $60 \mathrm{~min}$ and then centrifuged $(4500 \times g$, $3 \mathrm{~min})$. The phage-cell complexes from the pellets were placed on 300-mesh copper grids coated with carbon film and then stained with phosphotungstic acid $(2 \% \mathrm{w} / \mathrm{v}, \mathrm{pH}$ 7.2) for $30 \mathrm{~s}$. After drying, the preparation was examined by a transmission electron microscope.

\section{Genome sequencing and analysis}

The nucleic acid of phage ZZ1 was isolated as previously described [20]. Purified nucleic acid was used to determine susceptibility to DNase, RNase, and restriction enzymes and was then sent to Zhejiang California International NanoSystems Institute (Hangzhou, China) for commercial sequencing. The whole genome sequence, with a total length of $166,682 \mathrm{bp}$, was obtained using the Illumina Solexa Sequencing platform (Illumina, San Diego, USA) and the Swift analysis tool (http://swiftng. sourceforge.net) [30]. The genome sequence was analyzed with the NCBI BlastX bioinformatics tool (http://blast. ncbi.nlm.nih.gov/Blast.cgi) for nucleotide analysis, and the NCBI ORF finder (http://www.ncbi.nlm.nih.gov/projects/ gorf/) was used to identify ORFs, which were limited to those encoding proteins of greater than or equal to 50 amino acids. Homology assignments between genes from other phages and predicted ORFs of phage ZZ1 were based on amino acid sequence alignment searches (BlastP, http://blast.ncbi.nlm.nih.gov/Blast.cgi).

\section{Nucleotide sequence accession number}

The genome sequence, with a total length of 166,682 bp, for phage ZZ1 described in this work was submitted to GenBank and was assigned the accession number [GenBank: HQ698922].

\section{Competing interests}

The authors declare that they have no competing interests.

\section{Authors' contributions}

JJ conceived of the study and designed all the experiments and drafted the manuscript; ZJL, SWW, and DHH performed all phage-related experiments; SMW, YYM, and JW analyzed the clinical bacteria strains; FL and XDC participated in the TEM investigation; YHL, GXL, and XTW analyzed the phage genome. GQZ and ZQW participated in the design of the study and coordination and helped to draft the manuscript. All authors read and approved the final manuscript.

\section{Acknowledgements}

This study was supported by a Project of Open Research Fund Program of the State Key Laboratory of Virology of China (No. 2011007) and a Project of the Education Department of Henan Province (No. 2011 C310014).

\section{Author details}

${ }^{1}$ Department of Pathogen Biology, Basic Medical College of Zhengzhou University, Kexue Road \# 100, Zhengzhou 450001, P. R. China. ${ }^{2}$ Department of Pathogen Biology and Immunology, Henan Medical College for Staff and Workers, Zhengzhou, China. ${ }^{3}$ State Key Laboratory of Virology, Wuhan University, Wuhan, China. ${ }^{4}$ Clinical Laboratory, Henan Province People's Hospital, Zhengzhou, China.

Received: 11 January 2012 Accepted: 17 July 2012

Published: 28 July 2012

\section{References}

1. Barrow PA, Soothill JS: Bacteriophage therapy and prophylaxis: Rediscovery and renewed assessment of potential. Trends Microbiol 1997. 5:268-271

2. Carlton RM: Phage therapy: Past history and future prospects. Arch Immunol Ther Exp 1999, 47:267-274.

3. Merril C, Scholl D, Adhya SL: The prospect for bacteriophage therapy in Western medicine. Nat Rev Drug Discov 2003, 2:489-497. 
4. Garcia P, Monjardin C, Martin R, Madera C, Soberon N, Garcia E, Meana A, Suarez JE: Isolation of New Stenotrophomonas Bacteriophages and Genomic Characterization of Temperate Phage S1. Appl Environ Microbiol 2008, 74:7552-7560

5. Summers WC: Bacteriophage therapy. Annu Rev Microbiol 2001, 55:437-451.

6. Bruttin A, Brussow H: Human volunteers receiving Escherichia coli phage T4 orally: a safety test of phage therapy. Antimicrob Agents Chemother 2005, 49:2874-2878.

7. Capparelli R, Parlato M, Borriello G, Salvatore P, lannelli D: Experimental phage therapy against Staphylococcus aureus in mice. Antimicrob Agents Chemother 2007, 51:2765-2773.

8. Heo $Y$-J, Lee $Y-R$, Jung $H-H$, Lee J, Ko G, Cho Y-H: Antibacterial Efficacy of Phages against Pseudomonas aeruginosa Infections in Mice and Drosophila melanogaster. Antimicrob Agents Chemother 2009, 53:2469-2474

9. McVay CS, Velasquez M, Fralick JA: Phage therapy of Pseudomonas aeruginosa infection in a mouse burn wound model. Antimicrob Agents Chemother 2007, 51:1934-1938.

10. BergogneBerezin E, Towner KJ: Acinetobacter spp, as nosocomial pathogens: Microbiological, clinical, and epidemiological features. Clin Microbiol Rev 1996, 9:148-165.

11. Tsakris A, Pantazi A, Pournaras S, Maniatis A, Polyzou A, Sofianou D: Pseudooutbreak of imipenem-resistant Acinetobacter baumannii resulting from false susceptibility testing by a rapid automated system. Clin Microbiol 2000, 38:3505-3507.

12. Peleg AY, Seifert $H$, Paterson DL: Acinetobacter baumannii: Emergence of a successful pathogen. Clin Microbiol Rev 2008, 21:538-582.

13. Perez F, Hujer AM, Hujer KM, Decker BK, Rather PN, Bonomo RA: Global challenge of multidrug-resistant Acinetobacter baumannii. Antimicrob Agents Chemother 2007, 51:3471-3484.

14. Dijkshoorn L, Nemec A, Seifert H: An increasing threat in hospitals: multidrug-resistant Acinetobacter baumannii. Nat Rev Microbiol 2007, 5:939-951.

15. Navon-Venezia S, Ben-Ami R, Carmeli Y: Update on Pseudomonas aeruginosa and Acinetobacter baumannii infections in the healthcare setting. Curr Opin Infect Dis 2005, 18:306-313.

16. Ackermann HW, Brochu G, Konjin HPE: Classification Of Acinetobacter Phages. Arch Virol 1994, 135:345-354.

17. Klovins J, Overbeek GP, van den Worm SHE, Ackermann HW, van Duin J: Nucleotide sequence of a ssRNA phage from Acinetobacter: kinship to coliphages. J Gen Virol 2002, 83:1523-1533.

18. Petrov VM, Ratnayaka S, Nolan JM, Miller ES, Karam JD: Genomes of the T4related bacteriophages as windows on microbial genome evolution. Virol J 2010, 7:292.

19. Alisky J, Iczkowski K, Rapoport A, Troitsky N: Bacteriophages show promise as antimicrobial agents. J Infect 1998, 36:5-15.

20. Lin NT, Chiou PY, Chang KC, Chen LK, Lai MJ: Isolation and characterization of phi AB2: a novel bacteriophage of Acinetobacter baumannii. Res Microbiol 2010, 161:308-314.

21. Soothill JS: Treatment of experimental infections of mice with bacteriophages. J Med Microbiol 1992, 37:258-261.

22. Chibani-Chennoufi S, Bruttin A, Dillmann ML, Brussow H: Phage-host interaction: an ecological perspective. J Bacteriol 2004, 186:3677-3686.

23. Yang HJ, Liang L, Lin SX, Jia SR: Isolation and Characterization of a Virulent Bacteriophage AB1 of Acinetobacter baumannii. BMC Microbiol 2010, 10:10

24. Nakagawa T, Ishibashi II, Maruyama A, Yamanaka T, Morimoto Y, Kimura H, Urabe T, Fukui M: Analysis of dissimilatory sulfite reductase and $16 \mathrm{~S}$ rRNA gene fragments from deep-sea hydrothermal sites of the Suiyo Seamount, Izu-Bonin Arc, Western Pacific. Appl Environ Microbiol 2004, 70:393-403.

25. Adams MH: Bacteriophages. New York: Interscience; 1959.

26. Maniatis TSJ, Fritsch EF: Molecular Cloning: A Laboratory Manual. Cold Spring Harbor, NY: Spring Harbor Laboratory Press; 1982.

27. Jiang SC, Kellogg CA, Paul JH: Characterization of marine temperate phage-host systems isolated from Mamala Bay, Oahu, Hawaii. Appl Environ Microbiol 1998, 64:535-542.

28. Verma V, Harjai K, Chhibber S: Characterization of a T7-like lytic bacteriophage of Klebsiella pneumoniae B5055: a potential therapeutic agent. Curr Microbiol 2009, 59:274-281.
29. Capra ML, Quiberoni A, Reinheimer JA: Thermal and chemical resistance of Lactobacillus casei and Lactobacillus paracasei bacteriophages. Lett Appl Microbiol 2004, 38:499-504

30. Whiteford N, Skelly T, Curtis C, Ritchie ME, Lohr A, Zaranek AW, Abnizova I, Brown C: Swift: primary data analysis for the Illumina Solexa sequencing platform. Bioinformatics 2009, 25:2194-2199.

doi:10.1186/1471-2180-12-156

Cite this article as: Jin et al:: Isolation and characterization of ZZ1, a novel lytic phage that infects Acinetobacter baumannii clinical isolates. BMC Microbiology 2012 12:156.

\section{Submit your next manuscript to BioMed Central and take full advantage of:}

- Convenient online submission

- Thorough peer review

- No space constraints or color figure charges

- Immediate publication on acceptance

- Inclusion in PubMed, CAS, Scopus and Google Scholar

- Research which is freely available for redistribution

Submit your manuscript at www.biomedcentral.com/submit
C BioMed Central 